\title{
A BOUNDARY LAYER THEORY FOR SOME LINEAR AND NONLINEAR BOUNDARY VALUE PROBLEMS
}

\author{
F. A. HOWES * \\ Dedicated to Professor M. Nagumo \\ on the Occasion of his Seventieth Birthday
}

1. Introduction. In this note we consider some reformulations of a little known result of Brĩs [2] which relates the existence of a solution of the first order equation $f\left(t, u, u^{\prime}\right)=0$ to the existence and the asymptotic behavior (as $\epsilon \rightarrow 0^{+}$) of solutions of the boundary value problem

$$
\epsilon y^{\prime \prime}=f\left(t, y, y^{\prime}\right), a<t<b, y(a, \epsilon), y(b, \epsilon)
$$

prescribed. The theory developed here includes several earlier studies of problems of this form which possess turning points. Turning point problems can be characterized roughly as those whose reduced $(\epsilon=0)$ equations have singular points in $[a, b]$. Our principal technique of proof is Nagumo's theory of second order differential inequalities [13] (cf. also [10] for a more modern treatment) together with some elementary results from linear and nonlinear stability theory. The present discussion divides naturally into a consideration of two cases: namely, the quasilinear case, i.e., $f\left(t, y, y^{\prime}\right)=\mathrm{O}\left(\left|y^{\prime}\right|\right)$, as $\left|y^{\prime}\right| \rightarrow \infty$, and the quadratic case, i.e., $f\left(t, y, y^{\prime}\right)=\mathrm{O}\left(\left|y^{\prime}\right|^{2}\right)$, as $\left|y^{\prime}\right| \rightarrow \infty$. This follows on the one hand from the inherent limitation of Nagumo's theory to functions $f$ which are at most quadratic in $y^{\prime}$, and on the other, from the nonexistence of boundary layer behavior (in the solution itself) in the presence of cubic or higher nonlinearities with respect to $y^{\prime}$. (See, e.g., [17] and [16; Chap. 2].)

2. The Quasilinear Case. Consider now the boundary value problem

$$
\begin{gathered}
\epsilon y^{\prime \prime}=f\left(t, y, y^{\prime}\right), a<t<b, \\
y(a, \epsilon)=A, y(b, \epsilon)=B,
\end{gathered}
$$

together with the corresponding reduced equation

$$
f\left(t, u, u^{\prime}\right)=0, a<t<b .
$$

* Partially supported by the Mathematics Department of the University of Wisconsin at Madison, and by the National Science Foundation under grant no. MCS 76-05979. 
We study here and throughout the paper those reduced equations whose solutions $u$ need not satisfy either of the original boundary conditions (2.2), i.e., in general, $u(a) \neq A$ and $u(b) \neq B$. The following theorems are extensions of Theorem 4 in [2].

TheOREM 2.1. Assume

(1) the reduced equation (2.3) has a solution $u=u(t)$ which has a piecewise differentiable first derivative on $[a, b]$;

(2) the function $f$ is continuous in $\left(t, y, y^{\prime}\right)$, of class $C^{(2 q+1)}(q \geqq 0)$ with respect to $y$, and of class $C^{(1)}$ with respect to $y^{\prime}$ in $R$ :

$$
a \leqq t \leqq b,|y-u(t)| \leqq d_{1}(t, \epsilon),\left|y^{\prime}-u^{\prime}(t)\right| \leqq d_{2}(t, \epsilon),
$$

where

$$
\begin{aligned}
d_{1}(t, \epsilon)= & \mathrm{O}\left(|A-u(a)| \exp \left[-k \epsilon^{-1}(t-a)\right]\right) \\
& +\mathrm{O}\left(|B-u(b)| \exp \left[-k \epsilon^{-1}(b-t)\right]\right) \\
& +\mathrm{O}\left(\boldsymbol{\epsilon}^{(2 q+1)^{-1}}\right),
\end{aligned}
$$

and

$$
\begin{aligned}
d_{2}(t, \epsilon)= & \mathrm{O}\left(\boldsymbol{\epsilon}^{-1} \exp \left[-k \epsilon^{-1}(t-a)\right]\right) \\
& +\mathrm{O}\left(\boldsymbol{\epsilon}^{-1} \exp \left[-k \epsilon^{-1}(b-t)\right]\right) \\
& +\mathrm{O}\left(\boldsymbol{\epsilon}^{(2 q+1)^{-1}}\right) ;
\end{aligned}
$$

in addition, $f\left(t, y, y^{\prime}\right)=\mathrm{O}\left(\left|y^{\prime}\right|\right)$, as $\left|y^{\prime}\right| \rightarrow \infty,(t, y)$ in $R$;

(3) there exists a constant $k>0$ such that $f_{y^{\prime}} \leqq-k<0$ in $R \cap$ $[a, a+\delta]$, if $u(a) \neq A$, and $f_{y^{\prime}} \geqq k>0$ in $R \cap[b-\delta, b]$, if $u(b)$ $\neq B$, for $\delta$ a small positive constant;

(4) there exists a constant $m>0$ such that

$$
\partial_{y}{ }^{j} f\left(t, u(t), u^{\prime}(t)\right) \equiv 0,1 \leqq j \leqq 2 q, a \leqq t \leqq b,
$$

and

$$
\partial_{y}{ }^{2 q+1} f\left(t, y, u^{\prime}(t)\right) \geqq m>0,|y-u(t)| \leqq d_{1}(t, \epsilon), a \leqq t \leqq b .
$$

Then there exists an $\epsilon_{0}>0$ such that for each $\epsilon, 0<\epsilon \leqq \epsilon_{0}$, there exists a solution $y=y(t, \epsilon)$ of $(2.1),(2.2)$. Moreover, for $a \leqq t \leqq b$,

$$
\begin{aligned}
y(t, \epsilon)= & u(t)+\mathrm{O}\left(|A-u(a)| \exp \left[-k \epsilon^{-1}(t-a)\right]\right) \\
& +\mathrm{O}\left(|B-u(b)| \exp \left[-k \epsilon^{-1}(b-t)\right]\right) \\
& +\mathrm{O}\left(\epsilon^{(2 q+1)^{-1}}\right) .
\end{aligned}
$$


Proof. The theorem is proved by constructing lower and upper solutions of the full problem, i.e., functions $\alpha$ and $\beta$ which have piecewise differentiable first derivatives and which satisfy the inequalities: $\alpha \leqq \beta, \alpha(a, \epsilon) \leqq A \leqq \beta(a, \epsilon), \alpha(b, \epsilon) \leqq B \leqq \beta(b, \epsilon)$, and $\epsilon \alpha^{\prime \prime} \geqq f\left(t, \alpha, \alpha^{\prime}\right)$, $\epsilon \beta^{\prime \prime} \leqq f\left(t, \beta, \beta^{\prime}\right)$. Then, as follows from the theory in [13] or [10], the problem (2.1), (2.2) has a solution $y=y(t, \epsilon)$ for such $\epsilon>0$, with $\alpha(t, \epsilon) \leqq y(t, \epsilon) \leqq \beta(t, \epsilon), a \leqq t \leqq b$.

Suppose, for definiteness, that $u(a) \leqq A$ and $u(b) \geqq B$, then define for $\epsilon>0$ and $a \leqq t \leqq b$,

$$
\begin{aligned}
& \alpha(t, \epsilon)=u(t)-(u(b)-B) \exp \left[-k \epsilon^{-1}(b-t)\right] \\
& -\left(\epsilon \gamma m^{-1}\right)(2 q+1)^{-1}, \\
& \boldsymbol{\beta}(t, \boldsymbol{\epsilon})=\boldsymbol{u}(t)+(A-\boldsymbol{u}(a)) \exp \left[-k \boldsymbol{\epsilon}^{-1}(t-a)\right] \\
& +\left(\epsilon \boldsymbol{\gamma} \boldsymbol{m}^{-1}\right)(2 q+1)^{-1} \text {. }
\end{aligned}
$$

The constant $\gamma>0$ will be determined later. Clearly, $\alpha \leqq \beta, \alpha(a, \epsilon)$ $\leqq A \leqq \beta(a, \epsilon)$, and $\alpha(b, \epsilon) \leqq B \leqq \beta(b, \epsilon)$. To verify that the differential inequalities are satisfied, we first note that for $\sigma=\alpha$ or $\beta$,

$$
\begin{aligned}
f\left(t, \sigma, \sigma^{\prime}\right)= & f\left(t, u, u^{\prime}\right)+\left\{f\left(t, \sigma, u^{\prime}\right)-f\left(t, u, u^{\prime}\right)\right\} \\
& +\left\{f\left(t, \sigma, \sigma^{\prime}\right)-f\left(t, \sigma, u^{\prime}\right)\right\} \\
= & \sum_{j=1}^{2 q}(j !)^{-1} \partial_{y}{ }^{j} f\left(t, u, u^{\prime}\right)(\sigma-u)^{j} \\
& +((2 q+1) !)^{-1} \partial_{y}{ }^{2 q+1} f[\cdot](\sigma-u)^{2 q+1} \\
& +f_{y^{\prime}}[\cdots]\left(\sigma^{\prime}-u^{\prime}\right),
\end{aligned}
$$

where $[\cdot]=\left(t, u+\theta_{1}(\sigma-u), u^{\prime}\right)$ and $[\cdots]=\left(t, \sigma, u^{\prime}+\theta_{2}\left(\sigma^{\prime}-u^{\prime}\right)\right)$, $0<\theta_{1}, \theta_{2}<1$. Consider now only $\alpha=\alpha(t, \epsilon)$, since the demonstration for $\beta$ is analogous. Differentiating $\alpha$ and substituting into (2.1) via (2.4), we have by virtue of assumptions (1) and (4), for $E(t, \epsilon)=$ $(u(b)-B) \exp \left[-k \epsilon^{-1}(b-t)\right]$,

$$
\begin{aligned}
\epsilon \alpha^{\prime \prime}-f\left(t, \alpha, \alpha^{\prime}\right)= & \epsilon u^{\prime \prime}-k^{2} \epsilon^{-1} E(t, \epsilon) \\
& +((2 q+1) !)^{-1} \partial_{y}{ }^{2 q+1} f[\cdot]\{E(t, \epsilon) \\
& \left.+\left(\epsilon \gamma m^{-1}\right)^{(2 q+1)^{-1}}\right\}^{2 q+1} \\
& +f_{y^{\prime}}[\cdots] k \epsilon^{-1} E(t, \epsilon) \\
\geqq & -\epsilon M-k^{2} \epsilon^{-1} E(t, \epsilon)+\epsilon \gamma((2 q+1) !)^{-1} \\
& +f_{y^{\prime}}[\cdots] k \epsilon^{-1} E(t, \epsilon) .
\end{aligned}
$$


(Here $\left.\left|u^{\prime \prime}\right| \leqq M.\right)$ On the subinterval $[b-\delta, b]$, by assumption (3), $f_{y^{\prime}}[\cdots] \geqq k>0$; therefore, for such $t$,

$$
\begin{aligned}
\epsilon \alpha^{\prime \prime}-f\left(t, \alpha, \alpha^{\prime}\right) \geqq & -\epsilon M \pm k^{2} \epsilon^{-1} E(t, \epsilon) \\
& +\epsilon \gamma((2 q+1) !)^{-1} \\
\geqq & 0, \text { provided } \gamma \geqq(2 q+1) ! M .
\end{aligned}
$$

On the rest of the interval, $[a, b-\delta]$, the function $E(t, \epsilon)$ is transcendentally small, i.e., $E(t, \epsilon)=\mathrm{O}\left(\epsilon^{N}\right)$, for any integer $N \geqq 1$. Thus, for this range of $t$,

$$
\epsilon \alpha^{\prime \prime}-f\left(t, \alpha, \alpha^{\prime}\right) \geqq-\epsilon M+\epsilon \gamma((2 q+1) !)^{-1}-K \tau(\epsilon),
$$

where $K>0$ and $\tau(\epsilon)>0$ represents the contribution of the transcendentally small terms. For $\epsilon$ sufficiently small, we need only choose $\gamma=(2 q+1) !(M+1)$ to guarantee that the required inequality is satisfied on all of $[a, b]$. The existence of a solution $y=y(t, \epsilon)$ of $(2.1)$, (2.2) with $\alpha(t, \epsilon) \leqq y(t, \epsilon) \leqq \beta(t, \epsilon), a \leqq t \leqq b$, now follows from Nagumo's theorem. For the other cases in which $u(a) \neq A$ and $u(b) \neq B$, analogous bounding functions $\alpha$ and $\beta$ are defined.

We remark that this theorem is valid under the weaker assumptions that: (i) $\partial_{y}{ }^{j} f\left(t, u, u^{\prime}\right) \equiv 0,1 \leqq j \leqq 2 q, a+\delta \leqq t \leqq b-\delta$, and (ii) $\partial_{y}{ }^{2 q+1} f\left(t, u, u^{\prime}\right) \geqq m>0, a+\delta \leqq t \leqq b-\delta$. Briš [2] formulated this generalization in the case $q=0$. The proof of this only involves the construction of slightly more complicated bounding functions $\alpha$ and $\beta$. For example, suppose that $u(a)>A, u(b) \leqq B$ and $q=0$, i.e., $f_{y}\left(t, u, u^{\prime}\right) \geqq m>0, a+\delta \leqq t \leqq b$. Then define

$$
\alpha(t, \epsilon)=\left\{\begin{aligned}
u(t) & -(u(a)-A) \exp \left[\lambda_{1}(t-a)\right] \\
& -\epsilon \gamma \ell^{-1}\left(\exp \left[\lambda_{2}\left(t-t_{0}\right)\right]-1\right), \\
a & \leqq t \leqq t_{0}=a+\delta, \\
u(t) & -\epsilon \tilde{\gamma} m^{-1}-\lambda_{1}(u(a)-A) \exp \left[\lambda_{1}\left(t_{0}-a\right)\right] t \\
& +\epsilon \lambda_{2} \gamma \ell^{-1} \sigma^{-1} \exp \left[-\sigma\left(t-t_{0}\right)\right], \quad t_{0} \leqq t \leqq b .
\end{aligned}\right.
$$

Here $\lambda_{1}(\epsilon), \lambda_{2}(\epsilon)<0$ are the roots of $\epsilon \lambda^{2}+k \lambda+\ell=0$ of order $\mathrm{O}\left(\epsilon^{-1}\right), \mathrm{O}\left(\ell k^{-1}\right)$, respectively. The constant $\ell$ is a uniform bound on $\left|f_{y}\left(t, y, u^{\prime}\right)\right|, a \leqq t \leqq a+\delta,|y-u(t)| \leqq d_{1}(t, \epsilon)$. On each subinterval $[a, a+\delta]$ and $[a+\delta, b]$, this function satisfies the requisite inequalities, as follows by a direct computation, for $\sigma>0$ and sufficiently large.

In the next two theorems we present different formulations of assumption (4). 
Theorem 2.2. Assume (1), (2) and (3) as in Theorem 2.1 with the following exceptions: (i) $u^{\prime \prime} \geqq 0$ at those points in $[a+\delta, b-\delta]$ at which it exists, and (ii) $f$ is of class $C^{(n)}(n \geqq 2)$ with respect to $y$ in R. Assume also (4)' for $j=1, \cdots, n-1, \partial_{y}^{j} f\left(t, u(t), u^{\prime}(t)\right) \geqq 0, a+\delta$ $\leqq t \leqq b-\delta$; in addition, there exists a constant $m>0$ such that $\partial_{y}{ }^{n} f\left(t, u(t), u^{\prime}(t)\right) \geqq m>0, a+\delta \leqq t \leqq b-\delta$.

Then there exists an $\epsilon_{0}>0$ such that for each $\epsilon, 0<\epsilon \leqq \epsilon_{0}$, the problem (2.1), (2.2) has a solution $y=y(t, \epsilon)$ such that for $a \leqq t \leqq b$,

$$
\begin{aligned}
y(t, \epsilon)=u(t) & +\mathrm{O}\left(|A-u(a)| \exp \left[-k \epsilon^{-1}(t-a)\right]\right) \\
& +\mathrm{O}\left(|B-u(b)| \exp \left[-k \epsilon^{-1}(b-t)\right]\right) \\
& +\mathrm{O}\left(\epsilon^{n-1}\right) .
\end{aligned}
$$

TheOREM 2.3. Make the same assumptions as in Theorem 2.2 with the exceptions that in (1), $u^{\prime \prime} \leqq 0, a+\delta \leqq t \leqq b-\delta$, and that (4)' is replaced by (4)" for $j_{0, e}=1, \cdots, n-1 \partial_{y}{ }^{j_{0}} f\left(t, u(t), u^{\prime}(t)\right) \geqq 0$ and $\partial_{y}{ }^{j} f\left(t, u(t), u^{\prime}(t)\right) \leqq 0, a+\delta \leqq t \leqq b-\delta$, where $j_{0}$ denotes an odd integer and $j_{e}$ an even one; in addition, there exists a constant $m$ $<0$ such that $\partial_{y}{ }^{n} f\left(t, u(t), u^{\prime}(t)\right) \geqq m>0$, if $n$ is odd, or $\partial_{y}{ }^{n} f(t, u(t)$, $\left.u^{\prime}(t)\right) \leqq-m<0$, if $n$ is even, $a+\delta \leqq t \leqq b-\delta$.

Then the conclusion of Theorem 2.2 is valid.

The proofs of these theorems are similar to that of Theorem 2.1.

We consider next the case in which $f_{y^{\prime}}$ is only nonpositive in a neighborhood of $t=a$ and/or only nonnegative in a neighborhood of $t=b$.

THEOREM 2.4. Make the same assumptions as in Theorem 2.1 with the exception that assumption (3) is replaced by

$$
\begin{aligned}
& f_{y^{\prime}} \leqq 0, \text { in } R \cap[a, a+\delta], \text { if } u(a) \neq A, \text { and } \\
& f_{y^{\prime}} \geqq 0, \text { in } R \cap[b-\delta, b], \text { if } u(b) \neq B .
\end{aligned}
$$

Then there exists an $\epsilon_{0}>0$ such that for each $\epsilon, 0<\epsilon \leqq \epsilon_{0}$, there exists a solution $y=y(t, \epsilon)$ of $(2.1),(2.2)$. In addition, for $q=0$,

$$
\begin{aligned}
y(t, \epsilon)=u(t) & +\mathrm{O}\left(|A-u(a)| \exp \left[-\left(m \epsilon^{-1}\right)^{1 / 2}(t-a)\right]\right) \\
& +\mathrm{O}\left(|B-u(b)| \exp \left[-\left(m \epsilon^{-1}\right)^{1 / 2}(b-t)\right]\right) \\
& +\mathrm{O}(\epsilon), a \leqq t \leqq b ;
\end{aligned}
$$

while, for $q \geqq 1$,

$$
\begin{aligned}
y(t, \epsilon)=u(t) & +\mathrm{O}\left(|A-u(a)|\left(1+\sigma_{1} \epsilon^{-1 / 2}(t-a)\right)^{-q^{-1}}\right) \\
& +\mathrm{O}\left(|B-u(b)|\left(1+\sigma_{2} \epsilon^{-1 / 2}(b-t)\right)-q^{-1}\right) \\
& +\mathrm{O}\left(\epsilon^{[2 q(2 q+1)]^{-1}}\right), a \leqq t \leqq b .
\end{aligned}
$$


Here $\sigma_{1}, \sigma_{2}$ are positive constants which depend on $q \geqq 1$.

Theorem 2.5. Assume (1), (2) and (3) as in Theorem 2.4 with the exception that in (1), $u^{\prime \prime} \geqq 0$ at those points in $[a, b]$ at which it exists, and that $f$ is of class $C^{(n)}(n \geqq 2)$ with respect to $y$ in $R$. Assume also that $u(a) \leqq A, u(b) \leqq B$ and $\partial_{y}{ }^{j} f\left(t, u(t), u^{\prime}(t)\right) \geqq 0,1 \leqq j \leqq n-1$, $a \leqq t \leqq b$. Finally assume that $\partial_{y}{ }^{n} f\left(t, y, u^{\prime}(t)\right) \geqq m>0, a \leqq t \leqq b$, $|y-u(t)| \leqq d_{1}(t, \epsilon)$, for a positive constant $m$.

Then there exists an $\epsilon_{0}>0$ such that for each $\epsilon, 0<\epsilon \leqq \epsilon_{0}$, there exists a solution $y=y(t, \epsilon)$ of (2.1), (2.2). In addition, for $a \leqq t \leqq b$,

$$
\begin{aligned}
u(t) \leqq y(t, \epsilon) \leqq u(t) & +(A-u(a))\left(1+\bar{\sigma}_{1} \epsilon^{-1 / 2}(t-a)\right)^{-2(n-1)^{-1}} \\
& +(B-u(b))\left(1+\bar{\sigma}_{2} \epsilon^{-1 / 2}(b-t)\right)^{-2(n-1)^{-1}} \\
& +\bar{\gamma} \epsilon^{[n(n-1)]^{-1}},
\end{aligned}
$$

where $\bar{\gamma}$ is a known positive constant, and $\bar{\sigma}_{1}, \bar{\sigma}_{2}$ are positive constants which depend on $n \geqq 2$.

We remark that the conclusion of Theorem 2.5 is valid if instead we assume that $u^{\prime \prime} \leqq 0$ and that $\partial_{y}{ }^{(\text {odd })} f[u] \geqq 0, \partial_{y}{ }^{(\text {even })} f[u] \leqq 0$, with $\partial_{y}{ }^{n} f\left(t, y, u^{\prime}\right) \geqq m>0$, if $n$ is odd, and $\partial_{y}{ }^{n} f\left(t, y, u^{\prime}\right) \leqq-m<0$, if $n$ is even. Of course, we must also have $u(a) \geqq A$ and $u(b) \geqq B$.

These theorems are proved in basically the same manner as the previous ones. For example, as regards Theorem 2.4, suppose $u(a)$ $\geqq A, u(b) \leqq B$, and $q \geqq 1$. Then define for $\epsilon>0$ and $a \leqq t \leqq b$,

$$
\begin{aligned}
& \boldsymbol{\alpha}(t, \epsilon)= u(t)-(u(a)-A)\left(1+\sigma_{1} \epsilon^{-1 / 2}(t-a)\right)^{-q^{-1}} \\
&-\left(\epsilon^{(2 q)^{-1}} \gamma m^{-1}\right)^{(2 q+1)^{-1}}, \\
& \beta(t, \epsilon)= u(t)+(B-u(b))\left(1+\sigma_{2} \epsilon^{-1 / 2}(b-t)\right)-q^{-1} \\
&+\left(\epsilon(2 q)^{-1} \gamma m^{-1}\right)(2 q+1)^{-1}, \\
& \text { where } \sigma_{1}=q\left[m\{(q+1)(2 q+1) !\}^{-1}\right]^{-1 / 2}|A-u(a)|^{q}, \\
& \sigma_{2}=q\left[m\{(q+1)(2 q+1) !\}^{-1}\right]^{-1 / 2}|B-u(b)|^{q}, \text { and }
\end{aligned}
$$

$\gamma$ is a positive constant to be chosen sufficiently large. In the case of $\beta$, we have for

$$
\begin{aligned}
F(t, \epsilon)= & (B-u(b))\left(1+\sigma_{2} \epsilon^{-1 / 2}(b-t)\right)^{-q^{-1}}, \mathrm{f}\left(t, \boldsymbol{\beta}, \boldsymbol{\beta}^{\prime}\right)-\boldsymbol{\epsilon} \boldsymbol{\beta}^{\prime \prime} \\
= & ((2 q+1) !)^{-1} \partial_{y}{ }^{2 q+1} f[\cdot]\{F(t, \epsilon) \\
& \left.+\left(\epsilon^{(2 q)^{-1}} \gamma m^{-1}\right)^{(2 q+1)^{-1}}\right\}^{(2 q+1)} \\
& +f_{y^{\prime}}[\cdots] F^{\prime}(t, \epsilon)-\epsilon u^{\prime \prime}
\end{aligned}
$$




$$
\begin{aligned}
- & \left(\sigma_{2} q^{-1}\right)^{2}(q+1)(B-u(b))\left(1+\sigma_{2} \epsilon^{-1 / 2}(b-t)\right)^{-q^{-1}}(2 q+1) \\
\geqq & m((2 q+1) !)^{-1}\{F(t, \epsilon)\}^{2 q+1} \\
& +\epsilon^{(2 q)^{-1}} \gamma((2 q+1) !)^{-1} \\
& +f_{y^{\prime}}[\cdots] F^{\prime}(t, \epsilon)-\epsilon M \\
& -m((2 q+1) !)^{-1}\{F(t, \epsilon)\}^{2 q+1}
\end{aligned}
$$

(by the choice of $\left.\sigma_{2}\right)$. On the subinterval $[b-\delta, b], f_{y^{\prime}}[\cdots] \geqq 0$ by assumption (3)', and thus (since $F^{\prime}(t, \epsilon) \geqq 0$ ),

$f\left(t, \beta, \beta^{\prime}\right)-\epsilon \beta^{\prime \prime} \geqq \epsilon(2 q)^{-1} \gamma((2 q+1) !)^{-1}-\epsilon M \geqq 0$, if $\gamma \geqq M(2 q+1) !$

and $0<\epsilon<1$. On the rest of the interval, $[a, b-\delta]$, the estimates $\left|F^{\prime}(t, \epsilon)\right| \leqq K \epsilon^{(2 q)^{-1}}$ and $\left|f_{y^{\prime}}[\cdots]\right| \leqq L$ imply that

$$
f\left(t, \beta, \beta^{\prime}\right)-\epsilon \beta^{\prime \prime} \geqq \epsilon^{(2 q)^{-1}} \gamma((2 q+1) !)^{-1}-\epsilon M-\epsilon^{(2 q)^{-1}} L K \geqq 0,
$$

if $\gamma \geqq L K(2 q+1)$ ! and $0<\epsilon<1$. Thus the desired inequality holds on all of $[a, b]$ by setting $\gamma=(M+L K)(2 q+1)$ !.

We consider finally a situation in which the solution $u$ of the reduced equation has the "wrong" stability at $t=a$ or $t=b$, in the sense that $f_{y^{\prime}}[u]>0$ near $t=a$ or $f_{y^{\prime}}[u]<0$ near $t=b$. It will however turn out that a solution of the full problem (for small $\epsilon>0$ ) remains in a neighborhood of such a $u$ provided that $\left|f_{y^{\prime}}[u]\right|$ is not too large near $t=a$ or $t=b$, and that the function $u$ is stable with respect to the linearized coefficients of $y$. The simplest instance of such a phenomenon is contained in the next theorem.

TheOREM 2.6. Assume (1) and (2) as in Theorem 2.1 with $q=0$ and $R$ replaced by $\tilde{R}: a \leqq t \leqq b,|y-u(t)| \leqq d(t, \epsilon),\left|y^{\prime}\right|<\infty$, where $d(t, \epsilon) \geqq|A-u(a)|, \quad a \leqq t \leqq a+\delta$ and $d(t, \epsilon) \geqq|B-u(b)|, \quad b-\delta$ $\leqq t \leqq b$. Assume also (3)" for $\left(t, y, y^{\prime}\right)$ in $\tilde{R} \cap\left[a, a+\epsilon^{1 / 4}\right], f_{y^{\prime}}\left(t, y, y^{\prime}\right)$ $\geqq 0$ and $f_{y^{\prime}}\left(t, y, y^{\prime}\right)=\mathrm{O}\left(\epsilon^{p}\right), p>1 / 2$, if $u(a) \neq A$; for $\left(t, y, y^{\prime}\right)$ in $\tilde{R} \cap\left[b-\epsilon^{1 / 4}, b\right], f_{y^{\prime}}\left(t, y, y^{\prime}\right) \leqq 0$ and $f_{y^{\prime}}\left(t, y, y^{\prime}\right)=\mathrm{O}\left(\epsilon^{p}\right)$, if $\boldsymbol{u}(b) \neq$ B. Assume finally that $f_{y}\left(t, y, u^{\prime}(t)\right) \geqq m>0, a \leqq t \leqq b,|y-u(t)| \leqq$ $d(t, \epsilon)$, for a positive constant $m$.

Then there exists an $\epsilon_{0}>0$ such that for each $\epsilon, 0<\epsilon \leqq \epsilon_{0}$, the problem (2.1), (2.2) has a solution $y=y(t, \epsilon)$ such that for $a \leqq t \leqq b$,

$$
\begin{aligned}
y(t, \epsilon)=u(t) & +\mathrm{O}\left(|A-u(a)| \exp \left[-\left(m_{1} \epsilon^{-1}\right)^{1 / 2}(t-a)\right]\right) \\
& +\mathrm{O}\left(|B-u(b)| \exp \left[-\left(m_{1} \epsilon^{-1}\right)^{1 / 2}(b-t)\right]\right) \\
& +\mathrm{O}(\epsilon),
\end{aligned}
$$

where $0<m_{1}<m$. 
The proof of this theorem is once again a direct application of Nagumo's theorem. For instance, suppose that $u(a)<A, u(b)>B$, then define

$$
\beta(t, \epsilon)=u(t)+(A-u(a)) \exp \left[-\left(m_{1} \epsilon^{-1}\right)^{1 / 2}(t-a)\right]+\epsilon \gamma m^{-1} .
$$

Differentiating $\beta$ and substituting, we have for

$$
\begin{aligned}
H(t, \boldsymbol{\epsilon})= & (A-u(a)) \exp \left[-\left(m_{1} \epsilon^{-1}\right)^{1 / 2}(t-a)\right], \\
f\left(t, \boldsymbol{\beta}, \boldsymbol{\beta}^{\prime}\right)-\epsilon \boldsymbol{\beta}^{\prime \prime}= & f_{y}[\cdot]\left\{H(t, \boldsymbol{\epsilon})+\epsilon \boldsymbol{\gamma} m^{-1}\right\} \\
& +f_{y^{\prime}}[\cdots] H^{\prime}(t, \boldsymbol{\epsilon})-\epsilon \boldsymbol{u}^{\prime \prime}-m_{1} H(t, \epsilon) .
\end{aligned}
$$

On the subinterval $\left[a, a+\epsilon^{1 / 4}\right]$, since $f_{y^{\prime}}=\mathrm{O}\left(\epsilon^{p}\right)$,

$$
\begin{aligned}
f\left(t, \beta, \beta^{\prime}\right)-\epsilon \beta^{\prime \prime} \geqq & m H(t, \epsilon)+\epsilon \gamma-K \sqrt{m_{1}} \epsilon^{p-1 / 2} H(t, \epsilon) \\
& -\epsilon M-m_{1} H(t, \epsilon) .
\end{aligned}
$$

Now for $0<m_{1}<m$ and $\epsilon$ sufficiently small, $m>m_{1}+K \sqrt{m_{1}} \epsilon^{p-1 / 2}$. (Here $\left|f_{y^{\prime}}\right| \leqq K \epsilon^{p}$.) Consequently, by choosing $\gamma=M$, we have the desired inequality. On the rest of the interval, $\left[a+\epsilon^{1 / 4}, b\right]$, note that $H(t, \epsilon)$ is transcendentally small. Thus by choosing $\gamma=M+1$, $f\left(t, \beta, \beta^{\prime}\right)-\epsilon \beta^{\prime \prime} \geqq \epsilon \gamma-\tau(\epsilon)-\epsilon M \geqq 0$, where $\tau(\epsilon)>0$ represents the contribution of the transcendentally small terms.

3. Some Applications. We present here several immediate applications of the theory discussed above to quasilinear singular perturbation problems of the form

$$
\begin{gathered}
\epsilon y^{\prime \prime}=f(t, y) y^{\prime}+g(t, y), a<t<b, \\
y(a, \epsilon)=A, y(b, \epsilon)=B .
\end{gathered}
$$

An enormous amount of attention has been paid to such problems; the basic theory is summarized, for instance, in [18, Chap. 10] and in [15, Chap. 5]. These books also contain many additional references to the literature.

Consider first the case when $f(t, y)$ behaves like an even power of $t$ with $a=-1$ and $b=1$. Although $f(t, y)$ vanishes at $t=0$, the solution $y=y(t, \epsilon)$ of (3.1), (3.2) experiences only boundary layer behavior at $t=-1$ or $t=1$, depending on the sign of $f(t, y)$ near these endpoints. More precisely, if $f(t, y)$ is positive for $t$ in $[-1,1]$ $-\{0\}$, then $y(t, \epsilon)$ exhibits boundary layer behavior at $t=1$; if $f$ is negative for such $t$, then there is a boundary layer near $t=-1$. Of course, the reduced equation must have a smooth solution $u$ which is stable and which satisfies the appropriate boundary condition. Such results are known in the linear case (see, e.g., [14]) under the condi- 
tion of first order $y$-stability, i.e., $g_{y}[u]>0$. Our theory naturally includes these considerations and extends their validity to higher order $y$-stability. In the quasilinear case there are results of Dorr [3] and Howes [5] which again depend on first order $y$-stability. (Actually Dorr considers only the case of (3.1), (3.2) for which $g \equiv 0$.) The present treatment not only extends these results but also provides sharper boundary layer estimates than those in [5].

The theory of $\$ 2$ can also be viewed as establishing a framework for the existence of some types of resonant turning point behavior. This problem has likewise attracted much attention recently; namely, the formulation of necessary and sufficient conditions for the occurrence of resonant and nonresonant behavior in solutions of (3.1), (3.2). We mention here only the basic results of Ackerberg and O'Malley [1] and Kreiss and Parter [12]. In many of these problems one is forced to choose a solution $u$ of the reduced equation corresponding to (3.1) which satisfies neither boundary condition. The theory above gives then one set of sufficient conditions for the existence of solutions of (3.1), (3.2) which are close to $u$ on $(a, b)$. Similar phenomena are known to occur in the absence of any form of $y$-stability in the linear case; see, e.g., [1], [14], and [12]. The more general nonlinear case is currently under investigation [8] .

As an example of such behavior in the linear case, consider the simple problem

$$
\begin{aligned}
\epsilon y^{\prime \prime} & =t y^{\prime}+y,-1<t<1, \\
y(-1, \epsilon) & =A \neq 0, y(1, \epsilon)=B \neq 0 .
\end{aligned}
$$

The only bounded solution of $t u^{\prime}+u=0$ on $(-1,1)$ is $u \equiv 0$ which fails to satisfy either boundary condition. However, by Theorem 2.1, this problem has a solution $y=y(t, \epsilon)$ (which is, in fact, unique) of the form, for $-1 \leqq t \leqq 1$,

$$
y(t, \epsilon)=A \exp \left[-k \epsilon^{-1}(1+t)\right]+B \exp \left[-k \epsilon^{-1}(1-t)\right]
$$

plus a transcendentally small term, where $0<k<1$. The essential feature which characterizes the observed behavior is that the coefficient of $y^{\prime}$, namely $t$, has the correct sign at each endpoint, and consequently, the reduced root $u \equiv 0$ is a stable attractor of the rapidly changing boundary layer functions.

4. Quadratically Nonlinear Problems. We consider now briefly the case when the righthand side $f\left(t, y, y^{\prime}\right)$ of $(2.1)$ satisfies $f\left(t, y, y^{\prime}\right)=$ $\mathrm{O}\left(\left|y^{\prime}\right|^{2}\right)$, as $\left|y^{\prime}\right| \rightarrow \infty$. A more complete discussion can be found in [6]. The theory developed in $\$ 2$ carries ner with little change to this class of problems. The essential distinction is that in the presence 
of a nonlinearity of the form $\left(y^{\prime}\right)^{2}$, the behavior of the solution inside of the boundary layer depends on the sign of the (linearized) coefficient of $\left(y^{\prime}\right)^{2}$. To fix the ideas, consider the illustrative special case

$$
\begin{gathered}
\epsilon y^{\prime \prime}=p(t, y)\left(y^{\prime}\right)^{2}+q\left(t, y, y^{\prime}\right),-1<t<1, \\
y(-1, \epsilon)=A, y(1, \epsilon)=B,
\end{gathered}
$$

where $q$ is an affine function of $y^{\prime}$. Inside a boundary layer, say, at $t=-1$, the derivative $y^{\prime}$ is unbounded as a function of $\epsilon$ and therefore, $\epsilon y^{\prime \prime}$ is closely approximated by $p(t, y)\left(y^{\prime}\right)^{2}$. If $p(t, y)$ is positive near $t=-1$, then $\epsilon y^{\prime \prime}$ is also positive there, i.e., the solution $y$ is convex inside the layer. Similarly, if $p(t, y)$ is negative near $t=-1$, then $y$ is concave. Consequently, if the reduced solution $u$ is to approximate $y$ to the edge of the boundary layer, it must satisfy $(A-u(-1)) p(-1$, $y)>0$, for all $y$ between $A$ and $u(-1)$. By restricting the reduced solutions in this way, the theory given above applies in this quadratically nonlinear case. More generally, we can assume that $(A-u(a)) f_{y^{\prime} y^{\prime}}>0$ near $t=a$, if $u(a) \neq A$, and $(B-u(b)) f_{y^{\prime} y^{\prime}}>0$ near $t=b$, if $u(b) \neq B$.

5. More General Reduced Solutions. One of our basic assumptions has been that the reduced solution possesses a piecewise differentiable first derivative. However, one frequently encounters reduced paths which are composed of several distinct solutions of the reduced equation which intersect at an angle, i.e., their slopes are unequal. The first study of such phenomena was, of course, the classic paper of Haber and Levinson [4] which considered a quite general class of nonlinear righthand sides $f\left(t, y, y^{\prime}\right)$. Their treatment however neglected the possible occurrence of boundary layer behavior. Not surprisingly, one can adapt the basic theory of Haber and Levinson to the situations treated above. Some results in this direction are given in [7]. The basic assumptions here can again be motivated by stability considerations. A bounded solution of the full problem is attracted to a stable solution of the reduced equation. Stability (or instability) is first determined by the sign of $f_{y^{\prime}}$ evaluated along the branch of the reduced path in question. At an interior point, say, $t_{0}$, a root $u_{L}$ entering $t_{0}$ from the left is stable if $f_{y^{\prime}}\left[u_{L}\right] \geqq 0, t_{0}-\delta \leqq t \leqq t_{0}$, while a root $u_{R}$ entering from the right is stable if $f_{y^{\prime}}\left[u_{R}\right] \leqq 0, t_{0} \leqq t$ $\leqq t_{0}+\delta$. If $f_{y^{\prime}}\left[u_{L}, u_{R}\right]=0$ at $t=t_{0}$, then the root must be stable with respect to $\partial_{y}{ }^{j} f$ in any one of the senses outlined above. It may even be possible for a root $u_{L}$ or $u_{R}$ to attract a solution $y(t, \epsilon)$ if $f_{y^{\prime}}$ has the "wrong" sign near $t=t_{0}$, but is sufficiently small in magnitude 
there. Some of the theory of this "contrary" stability is also considered in [7].

6. Some Examples. In conclusion we study several simple examples which illustrate and motivate the theory discussed above.

Example 6.1. Consider the problem

$$
\begin{aligned}
\epsilon y^{\prime \prime} & =-t^{2 m} y^{\prime}+\left(y-t^{2}\right)^{2 n-1}+2 t^{2 m+1},-1<t<1, \\
y(-1, \epsilon) & =A, y(1, \epsilon)=1,
\end{aligned}
$$

where $m, n \geqq 1$ are integers.

The reduced solution of interest is $u=t^{2}$. By Theorem 2.1 we conclude that for each $\epsilon>0, \epsilon$ sufficiently small, this problem has a solution $y=y(t, \epsilon)$ with

$$
\begin{aligned}
y(t, \epsilon)= & t^{2}+O\left(|A-1| \exp \left[-k \epsilon^{-1}(1+t)\right]\right) \\
& +O\left(\epsilon^{(2 n-1)^{-1}}\right),-1 \leqq t \leqq 1,
\end{aligned}
$$

for $0<k<1$.

EXAMPLe 6.2. Consider now

$$
\begin{aligned}
\epsilon y^{\prime \prime} & =-t^{2 m+1} y^{\prime}+y^{2 r}, 0<t<1, \\
y(0, \epsilon) & =A \geqq 0, y(1, \epsilon)=0,
\end{aligned}
$$

where $m \geqq 0$ and $r \geqq 1$ are integers. Here the reduced solution which satisfies our hypotheses is $u \equiv 0$. By Theorem 2.5 we deduce the existence of a solution $y=y(t, \epsilon)$ for each $\epsilon>0$, $\epsilon$ sufficiently small, satisfying

$$
\begin{aligned}
0 \leqq y(t, \epsilon) \leqq & A\left(1+\sigma_{1}(n) \epsilon^{-1 / 2} t\right)-2(2 r-1)^{-1} \\
& +\bar{\gamma} \epsilon^{[2 r(2 r-1)]^{-1}}, 0 \leqq t \leqq 1,
\end{aligned}
$$

for positive constants $\sigma_{1}(n)$ and $\bar{\gamma}$.

Example 6.3. Consider next

$$
\begin{aligned}
\epsilon y^{\prime \prime} & =t y^{\prime}+y^{2}-t^{2}-|t|,-1<t<1, \\
y(-1, \epsilon) & =A>1, y(1, \epsilon)=B>1,
\end{aligned}
$$

and look at the reduced path $u(t)=|t|,-1 \leqq t \leqq 1$. Since $f_{y^{\prime}}=t$, this function $u$ is stable with respect to boundary layer behavior at $t= \pm 1$; however, it has contrary $f_{y^{\prime}}$-stability at $t=0$. Nevertheless, since $f_{y^{\prime}}$ is small near $t=0$ a solution $y=y(t, \epsilon)$ of this problem exists for small $\epsilon>0$ and satisfies 


$$
\begin{aligned}
|t| \leqq y(t, \epsilon) \leqq & |t|+(A-1) \exp \left[-k \epsilon^{-1}(1+t)\right] \\
& +(B-1) \exp \left[-k \epsilon^{-1}(1-t)\right] \\
& +\mathrm{O}\left(\epsilon^{1 / 2}\right),-1 \leqq t \leqq 1
\end{aligned}
$$

for $0<k<1$.

Example 6.4. Consider finally a problem with a quadratic nonlinearity, namely

$$
\begin{aligned}
\epsilon y^{\prime \prime} & =\left(y^{\prime}\right)^{2}-2 t y^{\prime}+y,-1<t<1, \\
y(-1, \epsilon) & =A, y(1, \epsilon)=B .
\end{aligned}
$$

The reduced equation $(*) u=2 t u^{\prime}-\left(u^{\prime}\right)^{2}$ is a D'Alembert equation (cf., e.g., equation 1.381 in [11]). We focus attention on the two parabolas $u_{d}(t)=t^{2}$ and $u_{1}(t)=3 t^{2} / 4$, and on the degenerate parabola $u_{2}(t) \equiv 0$. Clearly $u_{1}$ and $u_{2}$ are solutions of $\left(^{*}\right)$, while $u_{d}$ is the $p$ discriminant locus, i.e., any solution $u=u(t)$ of $\left({ }^{*}\right)$ satisfies $u(t) \leqq t^{2}$, $-1 \leqq t \leqq 1$. This follows by writing

$$
u(t)=2 t u^{\prime}(t)-\left(u^{\prime}(t)\right)^{2}=-\left(u^{\prime}(t)-t\right)^{2}+t^{2} .
$$

This if $A, B>1$, there is no solution of $(*)$ which satisfies either boundary condition. However, $u_{1}(t)=3 t^{2} / 4$ is stable with respect to boundary layer behavior at $t= \pm 1$, since $f_{y^{\prime}}\left[u_{1}\right]=t$. In addition, $u_{1}(-1)<A, u_{1}(1)<B$, and $f_{y^{\prime} y^{\prime}} \equiv 2$. Consequently, for each $\epsilon>0$, $\epsilon$ sufficiently small, the full problem (with, in fact, $A, B \geqq 3 / 4$ ) has a unique solution $y=y(t, \epsilon)$ satisfying

$$
\begin{aligned}
3 t^{2} / 4 \leqq & y(t, \epsilon) \\
\leqq & 3 t^{2} / 4+(A-3 / 4) \exp \left[-k \epsilon^{-1}(1+t)\right] \\
& +(B-3 / 4) \exp \left[-k \epsilon^{-1}(1-t)\right]+2 \epsilon, \\
& -1 \leqq t \leqq 1,
\end{aligned}
$$

for $0<k<1$.

Suppose now that $A=0$ and $B>3 / 4$. Although $u_{1}$ is stable at $t=-1$, this solution has the wrong convexity at $t=-1$ in the sense that $f_{y^{\prime} y^{\prime}} \equiv 2>0$ requires that $u_{1}(-1)<A$, and we have $u_{1}(-1)=$ $3 / 4>A=0$. Nevertheless, the reduced solution $u_{2} \equiv 0$ satisfies $u_{2}(-1)=0$ and, in addition, $f_{y^{\prime}}[0]=-2 t$. Thus if we define

$$
\hat{u}(t)=\left\{\begin{array}{lr}
0, & -1 \leqq t \leqq 0 \\
3 t^{2} / 4, & 0 \leqq t \leqq 1,
\end{array}\right.
$$

then $\hat{u}$ is of class $C^{(2)}[\{-1,1\}-\{0\}]$. More essential is the fact that 
$\hat{u}$ is stable with respect to boundary layer behavior at $t=1$, since $f_{y^{\prime}}[\hat{u}(t)]=t>0$ near $t=1$. Thus the full problem (with $A=0$ and $B \geqq 3 / 4)$ has a unique solution $y=y(t, \epsilon)$ for each $\epsilon>0$ sufficiently small which satisfies

$$
\begin{aligned}
\hat{u}(t) \leqq y(t, \epsilon) \leqq & \hat{u}(t)+(B-3 / 4) \exp \left[-k \epsilon^{-1}(1-t)\right] \\
& +2 \epsilon,-1 \leqq t \leqq 1,
\end{aligned}
$$

for $0<k<1$.

There are several other interesting types of asymptotic behavior associated with this particular problem and related ones; a more complete discussion is given in [9].

Acknowledgment. The author wishes to thank Professor W. R. Wasow for inviting him to spend a year at Wisconsin. He also gratefully acknowledges the support of the National Science Foundation.

\section{REFERENCES}

1. R. C. Ackerberg and R. E. O’Malley, Jr., Boundary Layer Problems Exhibiting Resonance, Studies in Appl. Math. 49 (1970), 277-295.

2. N. I. Brǐs, On Boundary Value Problems for the Equation $\epsilon y^{\prime \prime}=$ $f\left(x, y, y^{\prime}\right)$ for Small $\epsilon$, Dokl. Akad. Nauk SSSR 95 (1954), 429-432.

3. F. W. Dorr, Some Examples of Singular Perturbation Problems with Turning Points, SIAM J. Math. Anal. 1 (1970), 141-146.

4. S. Haber and N. Levinson, A Boundary Value Problem for a Singularly Perturbed Differential Equation, Proc. Amer. Math. Soc. 6 (1955), 866-872.

5. F. A. Howes, Singularly Perturbed Nonlinear Boundary Value Problems with Turning Points, SIAM J. Math. Anal. 6 (1975), 644-660.

6. - Singularly Perturbed Nonlinear Boundary Value Problems with Turning Points, II, ibid., to appear.

7. _- Modified Haber-Levinson Crossings, Trans. AMS, to appear.

8. — Resonant Structures in Nonlinear Singular Perturbation Theory, in preparation.

9. _- Singularly perturbed nonlinear boundary value problems whose reduced equations have singular points, Studies in Appl. Math., to appear.

10. L. K. Jackson, Subfunctions and Second Order Ordinary Differential Inequalities, Advances in Math. 2 (1968), 307-363.

11. E. Kamke, Differentialgleichungen, Lösungsmethoden und Lösungen, vol. 1, Chelsea, New York, 1971.

12. H. O. Kreiss and S. V. Parter, Remarks on Singular Perturbations with Turning Points, SIAM J. Math. Anal. 5 (1974), 230-251.

13. M. Nagumo, Über die Differentialgleichung $y^{\prime \prime}=f\left(x, y, y^{\prime}\right)$, Proc. Phys. Math. Soc. Japan 19 (1937), 861-866.

14. R. E. O’Malley, Jr., On Boundary Value Problems for a Singularly Perturbed Differential Equation with a Turning Point, SIAM J. Math. Anal. 1 (1970), 479-490. 
15. _—_, Introduction to Singular Perturbations, Academic Press, New York, 1974.

16. A. B. Vasil'eva, Asymptotic Behavior of Solutions to Certain Problems Involving Nonlinear Differential Equations Containing a Small Parameter Multiplying the Highest Derivatives, Russian Math. Surveys 18 (1963) 13-84.

17. M. I. Vishik and L. A. Lyusternik, Initial Jump for Nonlinear Differential Equations Containing a Small Parameter, Sov. Math. Dokl. 1 (1960), 749752.

18. W. R. Wasow, Asymptotic Expansions for Ordinary Differential Equations, Interscience, New York, 1965.

School of Mathematics, University of Minnesota, MinNeapolis, MN 55455 